\title{
Parental ADHD: Relations to Parenting, Child Behavior, and Treatment Outcomes
}

\author{
Charlotte Johnston $^{1}$ - Andrea Chronis-Tuscano ${ }^{2}$
}

Published online: 8 February 2017

(C) Springer Science+Business Media New York 2017

As editors, we are pleased to introduce this set of seven papers that form a special section devoted to the study of parental attention-deficit/hyperactivity disorder (ADHD). The idea for this section grew out of discussions of the work being conducted in our own labs and others focused broadly on families of children with ADHD. Research in this area traces a developmental trajectory from early studies that examined crosssectional relations between parenting and child ADHD, to research that distinguished between child ADHD versus child oppositional/conduct problems and that examined common parental psychopathologies such as depression and antisocial behavior, allowing us to isolate relations between these factors and parenting quality. As the heritability of ADHD became firmly established and the lifespan nature of the disorder was increasingly recognized, the most recent arc of this research trajectory reflects studies that place ADHD symptoms in the parent in a central role. Moreover, recent longitudinal research has begun to shed light on the impact of parental ADHD (alone or in combination with other factors) on the trajectory of child functioning over time, in line with the developmental psychopathology framework and our model of ADHD in families (Johnston and Chronis-Tuscano 2015). This special section was conceived as a way to bring together a compilation of these newer studies of parental ADHD to highlight similarities as well as differences in the emerging findings, to refine the models linking parental and child ADHD, and to spur further

Charlotte Johnston

cjohnston@psych.ubc.ca

1 Department of Psychology, 2136 West Mall, University of British Columbia, V6T 1Z4, Vancouver, BC, Canada

2 University of Maryland, College Park, MD, USA empirical tests of such models and to ultimately inform interventions to enhance child functioning.

As illustrated in Fig. 1, we conceive of parental ADHD, not only as conveying to the child a genetic risk for the disorder, but also as an important component of the parenting or environmental context that may alter the emergence and development of childhood ADHD and comorbid conditions, either directly or in interaction with genetic liability. The Figure serves only as a heuristic. It contains only some of the family, parent, and child characteristics that are likely functional in determining parenting and child outcomes (e.g., epigenetic effects are not explicitly noted in the figure nor are the influences of pre or perinatal risk factors) and that are examined in this special section. Not only are there acknowledged factors that are not included in this heuristic model, but the number and types of arrows that would be needed to illustrate the multiple, complex, and transactional influences among all of these parent, child and family variables is mindboggling (e.g., the possible gene $\mathrm{X}$ gene interactions, the impact of cultural norms on phenotypic expressions of ADHD). However, the Figure does, hopefully, convey a sense of the many possible relations among these variables and, most importantly, showcases the central role of parental ADHD symptoms that is the focus of this special section.

We invited and were fortunate to receive a set of papers that together present a comprehensive picture of the current state of the research. These papers cover a range of important aspects of parental ADHD and explore its linkages to various domains of family and child functioning. The research questions, samples, and measures employed across the studies reflect both commonalities and differences, which encourages consideration of the parameters that may be important in explaining the similarities and discrepancies in patterns of findings. Across studies, parental ADHD is examined as a correlate of parenting and/or child ADHD and externalizing 


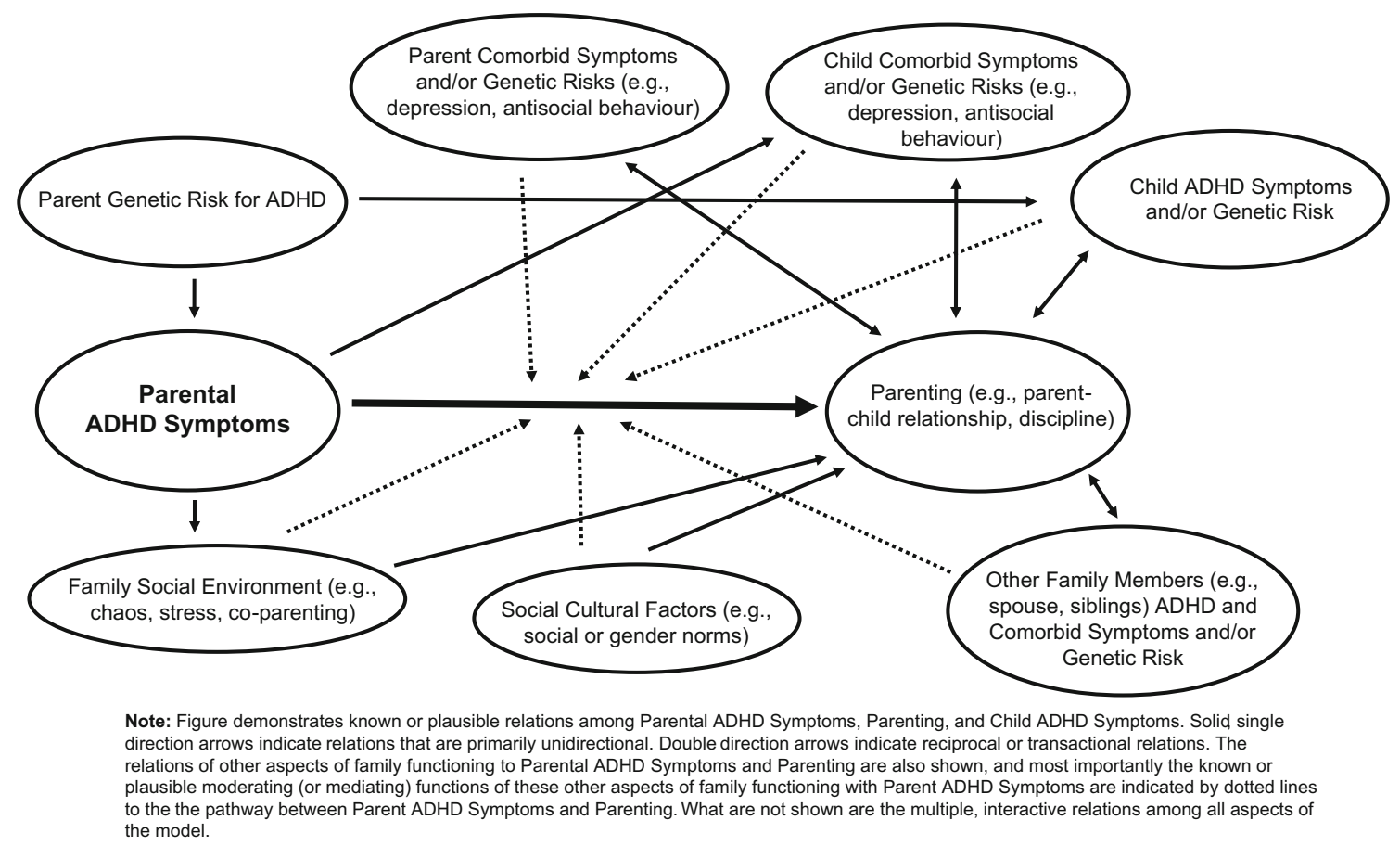

Fig. 1 A heuristic model of the relations between parental ADHD symptoms and other aspects of parent, child, and family functioning

problems, both concurrently and longitudinally, using multiple methods of assessment, and both correlational and experimental designs. In the longitudinal studies, parental ADHD is considered as both a static and dynamic predictor. In several of the studies, factors that may moderate or mediate the links between parental ADHD and child functioning, particularly genetic liabilities and parenting behaviors, are examined. The samples include children from infancy through to early adolescence, incorporating both comparison children/parents and children/parents with ADHD. Studies vary in whether one or both parents in a family are included, and whether parental ADHD is considered independently or interactively in mothers and fathers and/or children. Across studies, ADHD is considered both as a diagnostic category and dimensionally (with some studies focusing on the inattentive and hyperactive/impulsive symptom dimensions separately), and the role of other child and parent psychopathologies (particularly child oppositional defiant/conduct disorder and parental depression) are included. Beyond ADHD symptoms, most studies measure parenting behavior using questionnaire and/ or observational methodologies, and measures included across studies tap an intriguing range of family characteristics including parental neurocognitive functioning, family adversity, household chaos, co-parenting, and marital functioning.

Reflecting the known genetic nature of ADHD, two papers (Auerbach et al. 2017; Nikolas and Momany 2017) were solicited which incorporate the contributions of both genetic and environmental factors and their interaction in understanding parental ADHD in relation to child ADHD. Three papers examine parental ADHD and/or depression in relation to parenting or child outcomes. These papers examine longitudinal relations for mothers and fathers separately (Breaux et al. 2017), consider how mother and father symptom levels may interact (i.e., match/mismatch; Williamson et al. 2017), and explore three-way parent-partner-child interactions for behavior in triadic exchanges (Wymbs et al. 2017). One paper (Moroney et al. 2017) examines dynamic changes in parental and child ADHD symptoms over time, and the role of parenting behavior as a mediator of these changes. The last paper in the section (ChronisTuscano et al. 2017) reviews the literature on the impact of parental ADHD on outcomes of evidence-based child ADHD treatments and recent efforts to incorporate treatment of parental ADHD, discussing potential moderators of these effects, and summarizing ongoing work on how to best personalize and sequence ADHD treatments for parents and children.

Finally, we are delighted that Dr. Deater-Deckard agreed to serve as invited discussant for the special section. His comments, grounded in his own sophisticated and genetically-informed work on the underpinnings of parenting, place the papers in the context of the broader literature. His commentary provides a framework for seeing the convergent strengths of the work of all seven groups of authors. The commentary also provides a clear road map pointing to useful new methodologies and research foci for future studies of ADHD in families, thus completing our desire for this special section to serve as a catalyst for more and better research that will continue the upward trajectory of this research. 
In conclusion, we thank the authors and Dr. DeaterDeckard for their thoughtful contributions to this special section. We are excited to present this special section and trust that readers will agree with our assessment of the many strengths of the papers, both individually and collectively. We are hopeful that this special section will stimulate further research and that ultimately the prevention and intervention implications of this work will be realized to the benefit of families struggling with ADHD.

\section{Compliance with Ethical Standards}

Conflict of Interest The authors declare that they have no financial conflicts of interest.

Funding The first author acknowledges funding from the Canadian Institutes of Health Research CIHR 2010 MOP 106586.

The second author acknowledges funding from the National Institutes of Health (NIH R03 MH070666-1, R34 MH073567-01A1, R34 MH099208-01) and McNeil Pediatrics to study issues related to parental ADHD.

\section{References}

Auerbach, J. G., Zilberman-Hayun, Y., Atzaba-Poria, N., \& Berger, A. (2017). The contribution of maternal ADHD symptomatology, maternal DAT1, and home atmosphere to child ADHD symptomatol- ogy at 7 years of age. Journal of Abnormal Child Psychology. doi:10.1007/s10802-016-0230-0.

Breaux, R. P., Brown, H. R., \& Harvey, E. A. (2017). Mediators and moderators of the relation between parental ADHD symptomatology and the early development of child ADHD and ODD symptoms. Journal of Abnormal Child Psychology. doi:10.1007/s10802-0160213-1.

Chronis-Tuscano, A., Wang, C. H., Woods, K. E., Strickland, J., \& Stein, M. A. (2017). Parent ADHD and evidence-based treatment for their children: review and directions for future research. Journal of Abnormal Child Psychology. doi:10.1007/s10802-016-0238-5.

Johnston, C. \& Chronis-Tuscano, A. (2015). Families and ADHD. In R.A. Barkley (Ed.), Attention-deficit hyperactivity disorder: A handbook for diagnosis and treatment (pp.191-209). New York: Guilford.

Moroney, E., Tung, I., Brammer, W. A., Peris, T. S., \& Lee, S. S. (2017). Externalizing outcomes of youth with and without ADHD: timevarying prediction by parental ADHD and mediated effects. Journal of Abnormal Child Psychology. doi:10.1007/s10802-0160215-z.

Nikolas, M., \& Momany, A. (2017) DRD4 variants moderate the impact of parental characteristics on child attention-deficit hyperactivity disorder: exploratory evidence from a multiplex family design. Journal of Abnormal Child Psychology. doi:10.1007/s10802-0170264-y.

Williamson, D., Johnston, C., Noyes, A., Stewart, K., \& Weiss, M. (2017). Attention-deficit/hyperactivity disorder symptoms in mothers and fathers: family level interactions in relation to parenting. Journal of Abnormal Child Psychology. doi:10.1007/s10802016-0235-8.

Wymbs, B. T., Dawson, A. E., Egan, T. E., Sacchetti, G. M., Tams, S. T., $\&$ Wymbs, F. A. (2017). ADHD and depression symptoms in parent couples predict response to child ADHD and ODD behavior. Journal of Abnormal Child Psychology. doi:10.1007/s10802-0160220-2. 